\title{
Addition of fentanyl to the ultrasound-guided transversus abdominis plane block does not improve analgesia following cesarean delivery
}

\author{
LI-ZHONG WANG $^{1}$, XIA LIU ${ }^{2}$, YING-FA ZHANG ${ }^{1}$, XIAO-XIA HU ${ }^{1}$ and XIAO-MING ZHANG ${ }^{1}$ \\ Departments of ${ }^{1}$ Anesthesiology and ${ }^{2}$ Obstetrics, Jiaxing Maternity and Children Health Care Hospital, \\ Jiaxing, Zhejiang 314051, P.R. China
}

Received October 26, 2014; Accepted November 18, 2015

DOI: $10.3892 / \mathrm{etm} .2016 .3053$

\begin{abstract}
The present study aimed to investigate whether the addition of fentanyl to the transversus abdominis plane (TAP) block procedure may improve analgesic duration following cesarean delivery. A total of 147 nulliparous women with an American Society of Anesthesiologists physical status I-II, scheduled for elective cesarean delivery under spinal anesthesia, were enrolled in the present study. All patients underwent cesarean delivery under spinal anesthesia with $10 \mathrm{mg}$ bupivacaine and $10 \mu \mathrm{g}$ fentanyl, after which the patients underwent an ultrasound-guided bilateral TAP block with either $0.375 \%$ ropivacaine (group $\mathrm{T}_{\mathrm{R}} ; \mathrm{n}=48$ ), $0.375 \%$ ropivacaine and $50 \mu \mathrm{g}$ subcutaneous fentanyl (group $\mathrm{T}_{\mathrm{R}} \mathrm{S}_{\mathrm{F}} ; \mathrm{n}=49$ ), or a mixture of $0.375 \%$ ropivacaine and $50 \mu \mathrm{g}$ fentanyl $\left(2.5 \mu \mathrm{g} / \mathrm{ml}\right.$; group $\left.\mathrm{T}_{\mathrm{RF}} ; \mathrm{n}=50\right)$ per side. The TAP block formed part of a multimodal analgesic regimen comprising patient-controlled analgesia (PCA) with intravenous fentanyl, and regular treatment with diclofenac and paracetamol. The TAP block was performed in the midaxillary line using an in-plane technique. The primary outcome was the time to the first PCA, whereas secondary outcomes were the cumulative and interval PCA consumptions, visual analogue scale (VAS) pain scores at rest and during movement, side effects assessed at 2, 6, 12, 24 and $48 \mathrm{~h}$ postoperatively, and patient satisfaction with postoperative analgesia. No significant differences were observed in the median time to the first PCA among the three groups $(\mathrm{P}=0.640)$, which were $150 \mathrm{~min}$ (70-720 min) in group $T_{R}, 165 \mathrm{~min}(90-670 \mathrm{~min})$ in group $\mathrm{T}_{\mathrm{R}} \mathrm{S}_{\mathrm{F}}$, and $190 \mathrm{~min}$ (70-680 min) in group $\mathrm{T}_{\mathrm{RF}}$. Fentanyl consumption, VAS pain scores, side effects and patient satisfaction were similar among the three groups; however, the
\end{abstract}

Correspondence to: Mrs. Xia Liu, Department of Obstetrics, Jiaxing Maternity and Children Health Care Hospital, 2468 Zhong Huan Dong Road, Jiaxing, Zhejiang 314051, P.R. China

E-mail: liuxiazhejiang123@163.com

Key words: analgesia, obstetrical, adjuvants, anesthesia, fentanyl, nerve block demand for fentanyl was significantly decreased in the $T_{R} S_{F}$ and $\mathrm{T}_{\mathrm{RF}}$ groups at $2 \mathrm{~h}$ postoperatively $(\mathrm{P}=0.001$ and 0.002 , respectively), as compared with group $T_{R}$. No complications attributed to the TAP block were detected. In conclusion, the results of the present study suggested that the addition of $2.5 \mu \mathrm{g} / \mathrm{ml}$ fentanyl to the TAP block procedure was unable to improve analgesia following cesarean delivery under spinal anesthesia.

\section{Introduction}

Peripheral nerve blocks are a type of regional anesthesia. Local anesthetics (LAs) are injected near a specific nerve or bundle of nerves in order to block sensations of pain from a specific area of the body. Transversus abdominis plane (TAP) block is a newly described peripheral nerve block technique which can be used for any surgical technique that involves the lower abdominal wall $(1,2)$. TAP block can be performed by injecting LAs into the tissue plane between the internal oblique and the transversus abdominis via a blind technique, based on surface anatomy landmarks $(1,2)$ and, most recently, an ultrasound (US)-guided technique under direct vision $(3,4)$. TAP block has been shown to effectively provide analgesia, without the requirement for intrathecal morphine, as part of a multimodal analgesic regimen following cesarean delivery (5). However, the duration of analgesia provided by a single-injection TAP block has been demonstrated to be shorter, as compared with that of the intrathecal morphine (2-4).

There has been an interest in combining LAs with opioids to improve the duration and quality of peripheral nerve blocks, since the characterization of the opioid receptors in peripheral nerves $(6,7)$. Furthermore, various opioids, including fentanyl and sufentanil, have been shown to exert LA-like effects (8). However, the effects of opioids on peripheral nerve blocks are controversial; previous studies have reported both positive and negative effects for opioids (9-17). To the best of our knowledge, the use of opioids as analgesic adjuncts to the TAP block for postoperative analgesia are yet to be reported. The present study investigated whether the addition of fentanyl to an US-guided TAP block may prolong the analgesic duration following elective cesarean delivery 
under spinal anesthesia. In the present study, the systemic group, $\mathrm{T}_{\mathrm{R}} \mathrm{S}_{\mathrm{F}}$, was subcutaneously injected with fentanyl for comparison with the $\mathrm{T}_{\mathrm{RF}}$ group, in which fentanyl was injected into TAP. The aim of the present study was to investigate whether the analgesic effects of perineural fentanyl are local or systemic, as the majority of previous studies investigating the perineural application of fentanyl have not included a systemic control group (10-13).

\section{Materials and methods}

Study subjects. The present study was approved by the Institutional Ethics Committee of the Jiaxing Maternity and Children Health Care Hospital (Jiaxing, China). Written informed consent was obtained from all patients. A total of 150 nulliparous women ( $>37$ weeks) with an American Society of Anesthesiologists physical status I-II (18) treated at the Jiaxing Maternity and Children Health Care Hospital (Jiaxing, China) who were scheduled for elective cesarean delivery via a Pfannenstiel incision under spinal anesthesia, were enrolled in the present study between September 2013 and December 2013. Patients were excluded if they had a history of allergy to the drugs used in this study, contraindications to neuraxial anesthesia, tolerance to opioids or if they had used opioids within $48 \mathrm{~h}$ prior to the study. Three patients were excluded after randomization due to epidural supplementations; therefore, 147 patients were analyzed. In addition, patients with a body mass index of $>35 \mathrm{~kg} / \mathrm{m}^{2}$ were excluded.

US-guided TAP block procedure. No premedication was administered to the patients. Upon arrival at the operating room, a 20-gauge IV cannula (BD Biosciences, Franklin Lakes, NJ, USA) was inserted and standard monitoring was performed, including electrocardiography, blood oxygen saturation and non-invasive blood pressure measurements. Patients were randomly assigned to one of three groups, as follows: i) Group $T_{R}$, receiving TAP block with $0.375 \%$ ropivacaine (AstraZeneca, Södertälje, Sweden) $(n=48)$; ii) group $T_{R} S_{F}$, receiving TAP block with $0.375 \%$ ropivacaine plus $50 \mu \mathrm{g}$ subcutaneous fentanyl (Yichang Humanwell Pharmaceutical Co., Ltd., Yichang, China) $(\mathrm{n}=49)$; and iii) group $\mathrm{T}_{\mathrm{RF}}$, receiving TAP block with a mixture of $0.375 \%$ ropivacaine and $50 \mu \mathrm{g}$ fentanyl $(\mathrm{n}=50)$. Randomization was achieved using computer-generated codes created by SPSS 13.0 software (SPSS Inc., Chicago, IL, USA), which were maintained in sequentially numbered opaque envelopes prior to use. Spinal anesthesia was initiated in all patients in the left lateral position at the L2-3 interspace with $10 \mathrm{mg}$ isobaric bupivacaine (Shanghai Harvest Pharmaceutical Co., Ltd., Shanghai, China) and $10 \mu \mathrm{g}$ fentanyl, using a combined spinal-epidural technique (19). If the sensory level did not reach the T5 level after $15 \mathrm{~min}$, or the patient complained of pain intraoperatively, epidural supplementations of $2 \%$ lidocaine with epinephrine (1:200,000; Shanghai Hui Pharmaceutical Co., Ltd., Shanghai, China) were administered via an indwelling epidural catheter, and these patients were excluded from the present study.

Immediately following the completion of surgery and measurement of the residual block level, which assesses the level of dermatomal sensory block of spinal anesthesia following surgery via a pinprick, the patients underwent a US-guided bilateral TAP block with $19 \mathrm{ml}$ ropivacaine $(0.375 \%)$ and $1 \mathrm{ml}$ saline (groups $\mathrm{T}_{\mathrm{R}}$ and $\mathrm{T}_{\mathrm{R}} \mathrm{S}_{\mathrm{F}}$ ) or $1 \mathrm{ml}$ fentanyl (50 $\mu \mathrm{g}$; group $\mathrm{T}_{\mathrm{RF}}$ ) per side. The TAP block technique used was similar to the method outlined in a previous study (20). Briefly, with the patient in the supine position, an L38/10-5 MHz broadband linear array transducer (SonoSite, Inc., Bothell, WA, USA) was placed transversely onto the midaxillary line halfway between the iliac crest and the costal margin, and was adjusted in order to obtain the best possible image of the three muscle layers. A $100 \mathrm{~mm}$ 20-gauge short-bevel regional block needle (Zhejiang Sujia Medical Equipment Co., Ltd., Jiaxing, China) was introduced anteriorly through the skin, $3-4 \mathrm{~cm}$ medial to the US transducer, and was advanced into the fascial plane between the internal oblique muscle and the transversus abdominis muscle using an in-plane technique. The correct positioning of the needle tip was confirmed with injection of $1 \mathrm{ml}$ test dose, which consisted of $0.375 \%$ ropivacaine in group $T_{R}$ and $T_{R} S_{F}$, and a mixture of $0.375 \%$ ropivacaine and $50 \mu \mathrm{g}$ fentanyl in group $\mathrm{T}_{\mathrm{RF}}$, which resulted in separation between the internal oblique and transversus abdominis muscle. Subsequently, the remaining solution was injected in $5 \mathrm{ml}$ increments following careful aspiration under quantitative US guidance. Thereafter, the needle was withdrawn to the subcutaneous tissue, and the patients were subcutaneously administered with $1 \mathrm{ml}$ fentanyl $(50 \mu \mathrm{g})$ in the $\mathrm{T}_{\mathrm{R}} \mathrm{S}_{\mathrm{F}}$ group or $1 \mathrm{ml}$ saline in the $\mathrm{T}_{\mathrm{R}}$ and $\mathrm{T}_{\mathrm{RF}}$ groups. The same steps were repeated on the contralateral side. All solutions were prepared by a nurse anesthetist, who did not participate in the study, and all procedures were performed by a single operator using a sterile technique, including sterile gel and probe covers. The US images were evaluated by an independent investigator, in order to verify the needle placement and spreading of the solutions administered.

Postoperative treatment. All patients were treated with a routine postoperative analgesic regimen, consisting of $50 \mathrm{mg}$ oral diclofenac (Beijing Novartis Pharmaceutical Co., Ltd., Beijing, China) and $300 \mathrm{mg}$ rectal paracetamol (Northeast Pharmaceutical Co., Ltd., Shenyang, China) every $8 \mathrm{~h}$, combined with patient-controlled analgesia (PCA) with intravenous fentanyl (bolus dose, $30 \mu \mathrm{g}$; lockout interval, $10 \mathrm{~min}$ ) for $48 \mathrm{~h}$ postoperatively. The time to the first PCA was recorded as the analgesic duration of the TAP block. Patients were assessed at 2, 6, 12, 24 and $48 \mathrm{~h}$ postoperatively by an investigator blinded to the group allocation. During each assessment, cumulative and interval fentanyl consumptions, as well as the pain severity at rest and during movement (knee flexion), were recorded. The pain severity was quantified on a $10 \mathrm{~cm}$ visual analog scale (VAS) pain score (21), in which 0 indicated no pain and 10 indicated severe pain. In addition, the patients were evaluated for sedation, nausea, vomiting and complications that may have been associated with the TAP block. A 4-point scale was used to evaluate the level of sedation (1, fully awake; 2 , somnolent, responding to verbal stimuli; 3, somnolent, responding to tactile stimuli; and 4, somnolent, responding to painful stimuli), and nausea severity (1, none; 2, mild; 3, moderate; and 4, severe). An intravenous drip administering $4 \mathrm{mg}$ ondansetron was used in cases of reported nausea and/or vomiting. At $48 \mathrm{~h}$ following surgery, the patients were asked to rate their overall satisfaction with the postopera- 
Table I. Patient characteristics, duration of surgery and the block level at the end of surgery.

\begin{tabular}{|c|c|c|c|c|}
\hline & Group $\mathrm{T}_{\mathrm{R}}(\mathrm{n}=48)$ & Group $\mathrm{T}_{\mathrm{R}} \mathrm{S}_{\mathrm{F}}(\mathrm{n}=49)$ & Group $\mathrm{T}_{\mathrm{RF}}(\mathrm{n}=50)$ & P-value \\
\hline Age, years & $27.0 \pm 3.4$ & $27.8 \pm 3.4$ & $27.2 \pm 4.1$ & 0.653 \\
\hline Weight, kg & $68.7 \pm 6.8$ & $67.9 \pm 6.5$ & $70.1 \pm 6.9$ & 0.473 \\
\hline Height, cm & $160.1 \pm 4.2$ & $159.2 \pm 3.6$ & $161.2 \pm 4.6$ & 0.189 \\
\hline Gestational age, weeks & $38.9 \pm 1.1$ & $38.7 \pm 1.6$ & $39.1 \pm 1.2$ & 0.438 \\
\hline Duration of surgery, min & $36.6 \pm 8.1$ & $37.5 \pm 6.8$ & $35.8 \pm 7.5$ & 0.120 \\
\hline Level of sensory block & T6 (4-9) & T6 (4-8) & T5 (4-9) & 0.345 \\
\hline
\end{tabular}

Data are presented as the mean \pm standard deviation or the median (range). Group $T_{R}, T A P$ block with ropivacaine; group $T_{R} S_{F}$, TAP block with ropivacaine plus subcutaneous fentanyl; group $\mathrm{T}_{\mathrm{RF}}$, $\mathrm{TAP}$ block with ropivacaine-fentanyl mixture. TAP, transversus abdominis plane.

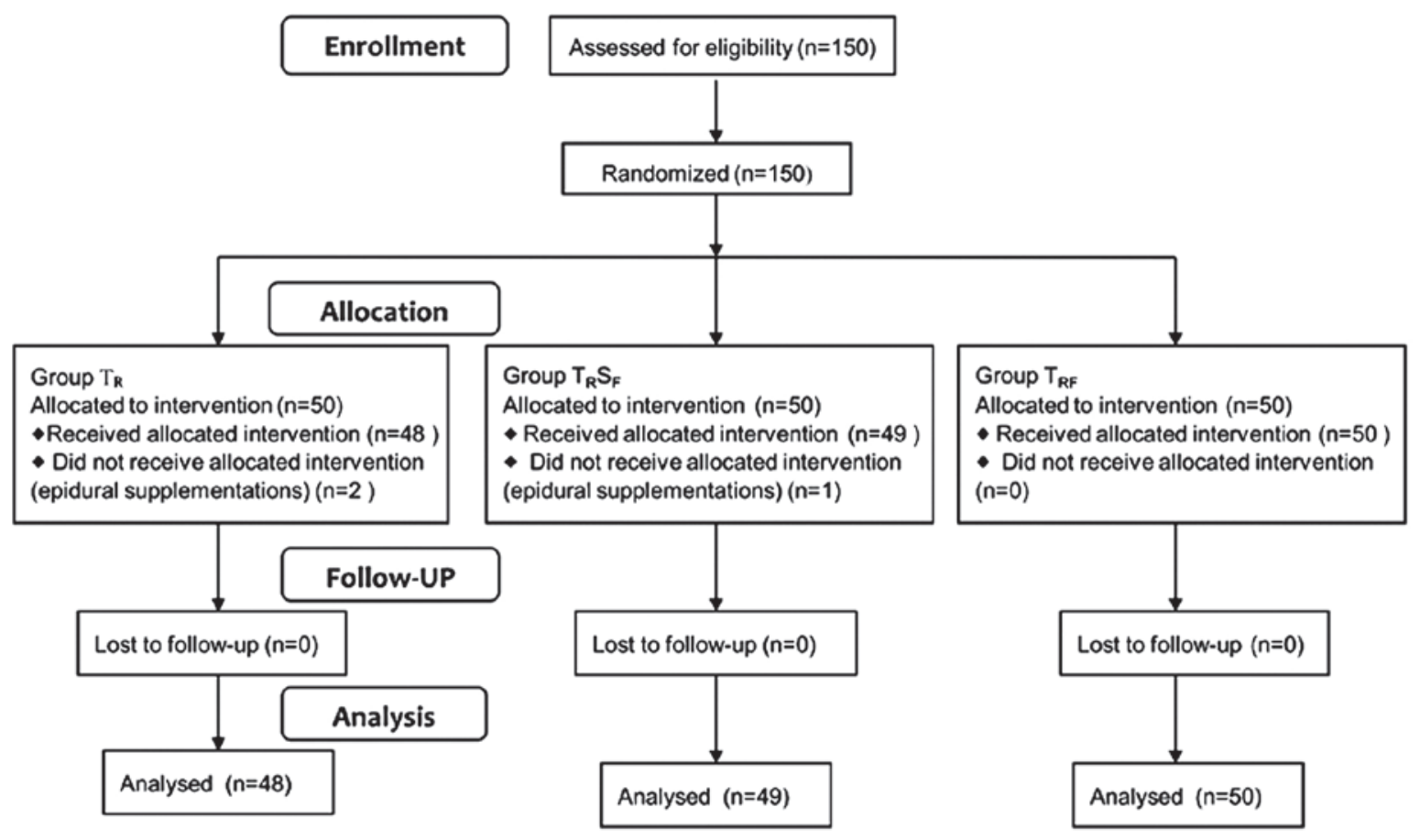

Figure 1. Flow diagram of patient distribution.

tive pain management on a 3-point scale: 1 , highly satisfied; 2 , satisfied; and 3, dissatisfied.

Statistical analysis. Data are presented as the mean \pm standard deviation, median (range) and count (\%), as appropriate. The primary outcome was the time to first PCA, in which the present study hypothesized that $\geq 60$ min differences between the three groups were clinically significant. Previous preliminary results obtained by the authors of the present study involving 10 patients demonstrated the duration of the TAP block with $0.375 \%$ ropivacaine alone was $140 \pm 80 \mathrm{~min}$; therefore, a sample size of $\geq 47$ patients per group was required, with an alpha risk of 0.05 and a power of 0.8 , to detect this difference between groups. In order to account for any drop-outs, a total of 50 patients were recruited for each group. The secondary outcome measures included the cumulative and interval PCA fentanyl consumptions, VAS pain scores at rest and during movement, side effects and patient satisfaction. The data were tested for normality using the Kolmogorov-Smirnov normality test. Continuous measurements were compared among the groups using repeated-measures or one-way analysis of variance if normally distributed, or Kruskal-Wallis $\mathrm{H}$ test if abnormally distributed. Categorical variables were compared using the the $\chi^{2}$ or Fisher's exact test, as appropriate. All statistical analyses were conducted using SPSS 13.0 software (SPSS, Inc.). The alpha level for all analyses was set as $\mathrm{P}<0.05$, and the Bonferroni correction for multiple comparisons was used where appropriate.

\section{Results}

Study subjects. A total of 150 patients were enrolled in the present study. Following randomization, 2 patients from group $T_{R}$ and 1 patient from group $T_{R} S_{F}$ were excluded due to requiring intraoperative epidural supplementations; thus, 147 patients were analyzed (Fig. 1). There were no statisti- 
Table II. Sedation, nausea, vomiting and patient satisfaction data.

\begin{tabular}{|c|c|c|c|c|}
\hline Parameter & Group $T_{R}(n=48)$ & Group $\mathrm{T}_{\mathrm{R}} \mathrm{S}_{\mathrm{F}}(\mathrm{n}=49)$ & Group $\mathrm{T}_{\mathrm{RF}}(\mathrm{n}=50)$ & P-value \\
\hline \multicolumn{5}{|l|}{ Sedation level } \\
\hline $2 \mathrm{~h}$ & $1(1-2)$ & $1(1-2)$ & $1(1-2)$ & 0.971 \\
\hline $6 \mathrm{~h}$ & $1(1-2)$ & $1(1-2)$ & $1(1-2)$ & 0.774 \\
\hline $12 \mathrm{~h}$ & $1(1-1)$ & $1(1-1)$ & $1(1-1)$ & 1.000 \\
\hline $24 \mathrm{~h}$ & $1(1-1)$ & $1(1-1)$ & $1(1-1)$ & 1.000 \\
\hline $48 \mathrm{~h}$ & $1(1-1)$ & $1(1-1)$ & $1(1-1)$ & 1.000 \\
\hline \multicolumn{5}{|l|}{ Nausea severity } \\
\hline $2 \mathrm{~h}$ & $1(1-3)$ & $1(1-3)$ & $1(1-3)$ & 0.352 \\
\hline $6 \mathrm{~h}$ & $1(1-3)$ & $1(1-3)$ & $1(1-2)$ & 0.654 \\
\hline $12 \mathrm{~h}$ & $1(1-2)$ & $1(1-2)$ & $1(1-2)$ & 0.621 \\
\hline $24 \mathrm{~h}$ & $1(1-2)$ & $1(1-2)$ & $1(1-2)$ & 0.923 \\
\hline $48 \mathrm{~h}$ & $1(1-2)$ & $1(1-2)$ & $1(1-2)$ & 0.857 \\
\hline $\begin{array}{l}\text { Number of patients } \\
\text { reporting vomiting, n (\%) }\end{array}$ & $7(14.5)$ & $9(18.3)$ & $11(22.0)$ & 0.638 \\
\hline Patient satisfaction & $1(1-3)$ & $1(1-3)$ & $1(1-2)$ & 0.502 \\
\hline
\end{tabular}

Data are presented as the median (range), unless otherwise stated. Group $T_{R}$, TAP block with ropivacaine; group $T_{R} S_{F}$, TAP block with ropivacaine plus subcutaneous fentanyl; group $\mathrm{T}_{\mathrm{RF}}$, TAP block with ropivacaine-fentanyl mixture. TAP, transversus abdominis plane.

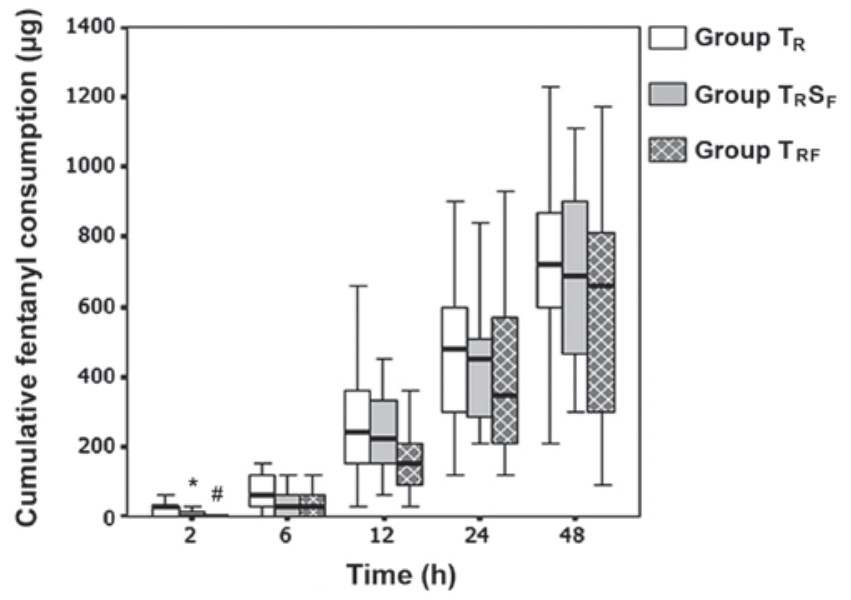

Figure 2. A box plot of the cumulative patient-controlled fentanyl consumptions for each group in the first $48 \mathrm{~h}$ postoperatively. Data are presented as the mean \pm standard deviation. Group $T_{R}$, TAP block with ropivacaine; group $\mathrm{T}_{\mathrm{R}} \mathrm{S}_{\mathrm{F}}$, TAP block with ropivacaine plus subcutaneous fentanyl; group $\mathrm{T}_{\mathrm{RF}}$, TAP block with ropivacaine and fentanyl mixture. TAP, transversus abdominis plane. ${ }^{*} \mathrm{P}=0.002 ;{ }^{\#} \mathrm{P}=0.001$ vs. group $\mathrm{T}_{\mathrm{R}}$.

cally significant differences between the groups in terms of patient characteristics, duration of surgery and the residual block level at the end of surgery (Table I). The TAP was easily localized by an US and blocks were uneventfully performed in all patients.

$P C A$. There was a tendency for earlier PCA in group $\mathrm{T}_{\mathrm{R}}$, as compared with the other two groups; however, the difference was not significantly different among the three groups $(\mathrm{P}=0.640)$. The median time to the first PCA was $150 \mathrm{~min}$ (range, 70-720 min) in group $\mathrm{T}_{\mathrm{R}}, 165 \mathrm{~min}$ (range, 90-670 min) in group $T_{R} S_{F}$, and $190 \mathrm{~min}$ (range, 70-680 min)

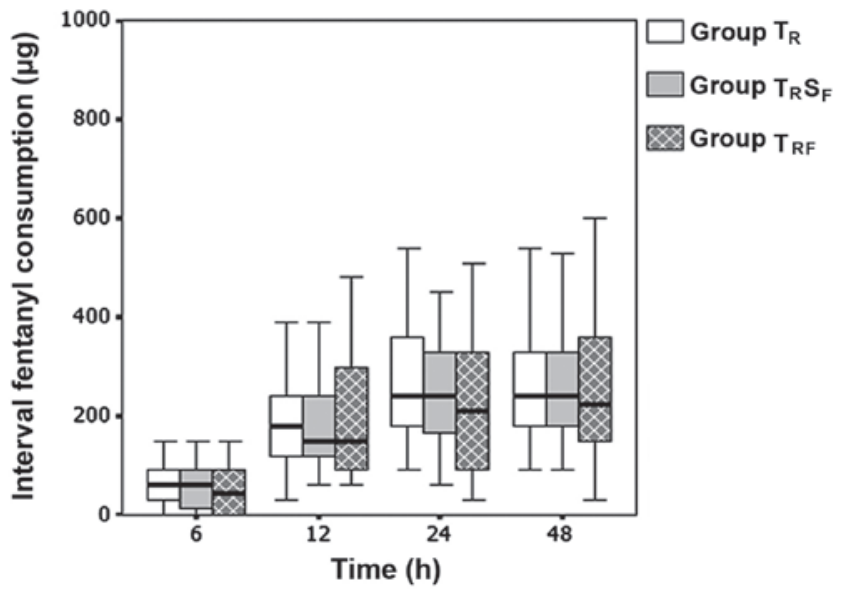

Figure 3. A box plot of the interval patient-controlled fentanyl consumptions for each group in the first $48 \mathrm{~h}$ postoperatively. Data are presented as the mean \pm standard deviation. Group $\mathrm{T}_{\mathrm{R}}$, TAP block with ropivacaine; group $\mathrm{T}_{\mathrm{R}} \mathrm{S}_{\mathrm{F}}$, TAP block with ropivacaine plus subcutaneous fentanyl; group $\mathrm{T}_{\mathrm{RF}}$, TAP block with ropivacaine-fentanyl mixture. TAP, transversus abdominis plane.

in group $\mathrm{T}_{\mathrm{RF}}$. Therefore, $2.5 \mu \mathrm{g} / \mathrm{ml}$ fentanyl, whether administered via TAP or subcutaneous route, was not capable of significantly prolonging the duration of analgesia, as compared with ropivacaine alone for TAP block.

Fentanyl consumption and VAS pain scores. No statistically significant differences were observed in the cumulative and interval fentanyl consumption (Figs. 2 and 3) and the VAS pain scores at rest and during movement (Figs. 4 and 5), among the three groups at all time points. This indicated that the postoperative analgesic quality was not improved when $2.5 \mu \mathrm{g} / \mathrm{ml}$ fentanyl was added to the TAP block. However, 


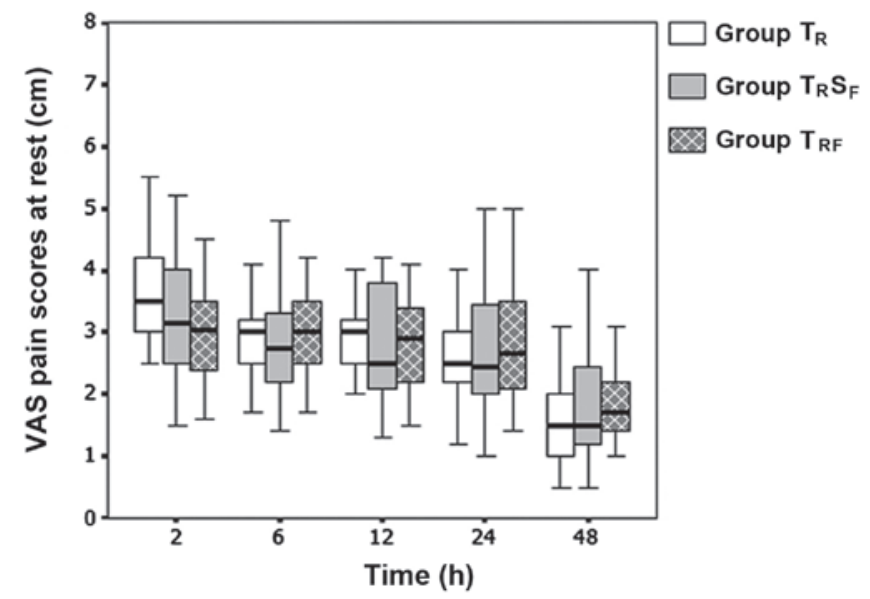

Figure 4. A box plot of the VAS pain scores at rest for each group in the first $48 \mathrm{~h}$ postoperatively. Data are presented as the mean \pm standard deviation. Group $T_{R}$, TAP block with ropivacaine; group $T_{R} S_{F}$, TAP block with ropivacaine plus subcutaneous fentanyl; group $\mathrm{T}_{\mathrm{RF}}$, TAP block with ropivacaine-fentanyl mixture. VAS, visual analog scale; TAP, transversus abdominis plane.

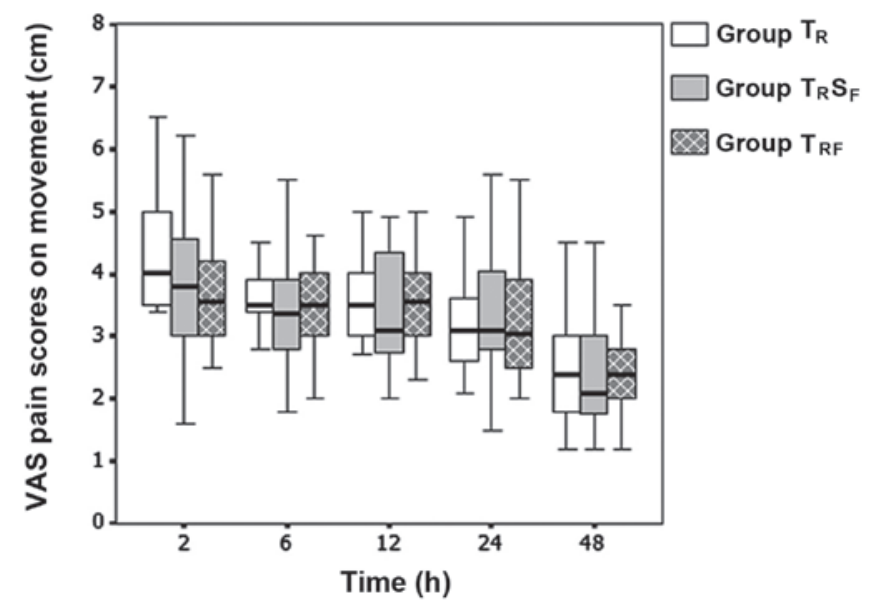

Figure 5. A box plot of the VAS pain scores during movement in each group in the first $48 \mathrm{~h}$ postoperatively. Data are presented as the mean \pm standard deviation. Group $T_{R}$, TAP block with ropivacaine; group $T_{R} S_{F}$, TAP block with ropivacaine plus subcutaneous fentanyl; group $\mathrm{T}_{\mathrm{RF}}$, TAP block with ropivacaine-fentanyl mixture. VAS, visual analog scale; TAP, transversus abdominis plane.

the cumulative fentanyl consumption was significantly lower in the $\mathrm{T}_{\mathrm{R}} \mathrm{S}_{\mathrm{F}}$ and $\mathrm{T}_{\mathrm{RF}}$ groups at $2 \mathrm{~h}$ postoperatively $(\mathrm{P}=0.002$ and 0.001 , respectively), as compared with that in group $T_{R}$. By contrast, no statistically significant difference in the cumulative fentanyl consumption was observed at this time point between the $T_{R} S_{F}$ and $T_{R F}$ groups ( $P=0.273$; Fig. 2). Therefore, the early analgesic-sparing effects following caesarean delivery in the TRSF and TRF groups may be explained by the systemic absorption of fentanyl. In addition, no significant differences were identified in the postoperative sedation and nausea levels, number of patients that reported vomiting, antiemetic requirements and patient satisfaction with postoperative analgesia, among the three groups (Table II). Furthermore, there were no complications, such as LA systemic toxicity, vascular or visceral injury, attributed to the TAP block in any of the groups.

\section{Discussion}

The results of the present study suggested that the addition of $2.5 \mu \mathrm{g} / \mathrm{ml}$ fentanyl to the TAP block procedure $(0.375 \%$ ropivacaine) was unable to improve the duration and quality of analgesia following caesarean delivery. In addition, as the cumulative fentanyl consumption was significantly lower in the $\mathrm{T}_{\mathrm{R}} \mathrm{S}_{\mathrm{F}}$ and $\mathrm{T}_{\mathrm{RF}}$ groups at $2 \mathrm{~h}$ postoperatively, as compared with that in group $T_{R}$, the early analgesic-sparing effect associated with perineural fentanyl may have been mediated by systemic effects due to peripheral uptake.

The TAP block is commonly used during a cesarean delivery, in which it forms part of a multimodal postoperative analgesic regimen (2-5). Previous studies have demonstrated that a single injection of TAP block was able to significantly improve post-cesarean analgesia in the absence of spinal morphine; however, the analgesic duration was limited, as compared with intrathecal morphine (2-4). Numerous analgesic adjuncts have been added to LAs in various peripheral nerve blocks in order to improve the analgesic duration (7); however, the addition of analgesic adjuncts to the TAP block has rarely been reported. A previous study has demonstrated that the addition of $8 \mathrm{mg}$ dexamethasone to $20 \mathrm{ml}$ bupivacaine $(0.25 \%)$ in the TAP block was associated with a longer time to the first PCA, lower postoperative pain scores and reduced morphine requirements in patients undergoing open abdominal hysterectomy (22). By contrast, Bollag et al (23) reported that the addition of $75 \mu \mathrm{g}$ clonidine to $0.375 \%$ bupivacaine in the TAP block did not result in improved pain scores following cesarean delivery.

The existence of opioid receptors at peripheral nerves and the LA-like action of various opioids, including fentanyl and sufentanil, have been demonstrated in vitro $(6,8)$; however, there has been controversy regarding the efficacy of the perineural application of opioids in clinical trials. Murphy et al (24) reported that opioids had limited benefits as analgesic adjuncts to the brachial plexus block, whereas they were shown to exert a definite but mild analgesia following intraarticular injection in another study (25). Fentanyl is an opioid that has been widely used as an analgesic adjunct to LAs in clinical practice. Previous studies have reported improved analgesia following the addition of fentanyl to LAs in various nerve blocks, including the axillary brachial plexus $(9,10)$, cervical plexus (11), infraorbital nerve (12), caudal block (13) and paravertebral analgesia (14). Conversely, other studies (15-17) have been unable to detect improved analgesia following co-administration of LAs and fentanyl. The conflicting findings of previous studies may be due to differences in the concentrations and doses used, as well as due to methodological differences in the study design, including a lack of systemic control groups, and the site and technique of nerve blocks. To the best of our knowledge, the present study is the first to investigate the effects of fentanyl addition to the TAP block on postoperative analgesia. A $2.5 \mu \mathrm{g} / \mathrm{ml}$ fentanyl dose (total dose, $100 \mu \mathrm{g}$ ) was selected in the present study, since previous studies have demonstrated that this concentration of fentanyl was able to significantly improve the effectiveness of peripheral nerve blocks $(9-11,14)$. Furthermore, this dose was selected due to concerns regarding potential dose-dependent adverse systemic effects associated with fentanyl. 
The present study demonstrated that the addition of $2.5 \mu \mathrm{g} / \mathrm{ml}$ fentanyl to ropivacaine was unable to affect the TAP block analgesia following cesarean delivery, as assessed by the duration of analgesia, VAS pain scores and post-surgery analgesic requirements. The reduced requirement for additional analgesia in the $T_{R} S_{F}$ and $T_{R F}$ groups during the first $2 \mathrm{~h}$ postoperation, as compared with the $T_{R}$ group, may have been due to the systemic effects of fentanyl, since no significant difference was detected between the $\mathrm{T}_{\mathrm{R}} \mathrm{S}_{\mathrm{F}}$ and $\mathrm{T}_{\mathrm{RF}}$ groups. The reasons why fentanyl was unable to improve the analgesia of the TAP block are unclear. One plausible explanation may be that fentanyl was unable to diffuse through the nerve sheath (8). In addition, the median time to first PCA was markedly shorter in the present study, as compared with those reported in previous studies, including $220 \mathrm{~min}$ (150-380 $\mathrm{min}$ ) (2), $3 \mathrm{~h}$ (inter-quartile range, $1 \mathrm{~h}$ ) (3) and $4 \mathrm{~h}$ (0.5-29 h) (4). This may be due to the higher doses of bupivacaine and fentanyl used for the spinal anesthesia, and the higher concentrations (and doses) of LAs used for the TAP block in previous studies (2-4), as compared with the present study.

A limitation of the present study was that the extent of abdominal wall sensory block following the TAP block was not assessed, due to a residual sensory block from spinal anesthesia in the early postoperative period; thus, the failed blocks were not excluded. However, the TAP blocks were conducted by an experienced operator, and the US approach allowed for direct visualization of the needle and of the placement of LAs, which should have reduced the risk of block failure. Another limitation was the relatively low concentration of fentanyl used, and the fact that other adjuncts, including epinephrine, were not assessed in the present study. Therefore, whether a higher concentration of fentanyl, or a combination of fentanyl and epinephrine, may improve the analgesic efficacy of the TAP block should be the focus of future studies. Large individual variations were detected in post-cesarean delivery analgesia in the present study, which may be associated with the characteristics of post-operative analgesia per se or an inadequate sample size, which may limit the conclusions of the present study.

In conclusion, the results of the present study suggested that the addition of $2.5 \mu \mathrm{g} / \mathrm{ml}$ fentanyl to $0.375 \%$ ropivacaine was unable to improve the TAP block analgesia following cesarean delivery under spinal anesthesia. Future studies should endeavour to investigate other measures, including the application of the continuous catheter-based technique, that may prolong the postoperative analgesic duration of the TAP block.

\section{References}

1. McDonnell JG, O’Donnell B, Curley G, Heffernan A, Power C and Laffey JG: The analgesic efficacy of transversus abdominis plane block after abdominal surgery: A prospective randomized controlled trial. Anesth Analg 104: 193-197, 2007.

2. McDonnell JG, Curley G, Carney J, Benton A, Costello J, Maharaj $\mathrm{CH}$ and Laffey JG: The analgesic efficacy of transversus abdominis plane block after cesarean delivery: A randomized controlled trial. Anesth Analg 106: 186-191, 2008.

3. Belavy D, Cowlishaw PJ, Howes M and Phillips F: Ultrasound-guided transversus abdominis plane block for analgesia after caesarean delivery. Br J Anaesth 103: 726-730, 2009 .
4. Kanazi GE, Aouad MT, Abdallah FW, Khatib MI, Adham AM, Harfoush DW and Siddik-Sayyid SM: The analgesic efficacy of subarachnoid morphine in comparison with ultrasound-guided transversus abdominis plane block after cesarean delivery: A randomized controlled trial. Anesth Analg 111: 475-481, 2010.

5. Abdallah FW, Halpern SH and Margarido CB: Transversus abdominis plane block for postoperative analgesia after caesarean delivery performed under spinal anaesthesia? A systematic review and meta-analysis. Br J Anaesth 109: 679-687, 2012.

6. Stein C and Lang LJ: Peripheral mechanisms of opioid analgesia. Curr Opin Pharmacol 9: 3-8, 2009.

7. Axelsson K and Gupta A: Local anaesthetic adjuvants: Neuraxial versus peripheral nerve block. Curr Opin Anaesthesiol 22: 649-654, 2009.

8. Gissen AJ, Gugino LD, Datta S, Miller J and Covino BG: Effects of fentanyl and sufentanil on peripheral mammalian nerves. Anesth Analg 66: 1272-1276, 1987.

9. Nishikawa K, Kanaya N, Nakayama M, Igarashi M, Tsunoda K and Namiki A: Fentanyl improves analgesia but prolongs the onset of axillary brachial plexus block by peripheral mechanism. Anesth Analg 91: 384-387, 2000.

10. Karakaya D, Büyükgöz F, Bariş $S$, Güldoğuş F and Tür A: Addition of fentanyl to bupivacaine prolongs anesthesia and analgesia in axillary brachial plexus block. Reg Anesth Pain Med 26: 434-438, 2001.

11. Sindjelic RP, Vlajkovic GP, Davidovic LB, Markovic DZ and Markovic MD: The addition of fentanyl to local anesthetics affects the quality and duration of cervical plexus block: A randomized, controlled trial. Anesth Analg 111: 234-237, 2010.

12. Mane RS, Sanikop CS, Dhulkhed VK and Gupta T: Comparison of bupivacaine alone and in combination with fentanyl or pethidine for bilateral infraorbital nerve block for postoperative analgesia in pediatric patients for cleft lip repair: A prospective randomized double blind study. J Anaesthesiol Clin Pharmacol 27: 23-26, 2011.

13. Constant I, Gall O, Gouyet L, Chauvin M and Murat I: Addition of clonidine or fentanyl to local anaesthetics prolongs the duration of surgical analgesia after single shot caudal block in children. Br J Anaesth 80: 294-298, 1998.

14. Burlacu CL, Frizelle HP, Moriarty DC and Buggy DJ: Fentanyl and clonidine as adjunctive analgesics with levobupivacaine in paravertebral analgesia for breast surgery. Anaesthesia 61: 932-937, 2006.

15. Fanelli G, Casati A, Magistris L, Berti M, Albertin A, Scarioni M and Torri G: Fentanyl does not improve the nerve block characteristics of axillary brachial plexus anaesthesia performed with ropivacaine. Acta Anaesthesiol Scand 45: 590-594, 2001.

16. Fletcher D, Kuhlman G and Samii K: Addition of fentanyl to $1.5 \%$ lidocaine does not increase the success of axillary plexus block. Reg Anesth 19: 183-188, 1994.

17. Johansson A, Kornfält J, Nordin L, Svensson L, Ingvar C and Lundberg J: Wound infiltration with ropivacaine and fentanyl: Effects on postoperative pain and PONV after breast surgery. J Clin Anesth 15: 113-118, 2003.

18. American Society of Anesthesiologists: New classification of physical status. Anesthesiology 24: 111, 1963.

19. Birnbach DJ and Ojea LS: Combined spinal-epidural (CSE) for labor and delivery. Int Anesthesiol Clin 40: 27-48, 2002.

20. Hebbard P, Fujiwara Y, Shibata Y and Royse C: Ultrasound-guided transversus abdominis plane (TAP) block. Anaesth Intensive Care 35: 616-617, 2007.

21. DeLoach LJ, Higgins MS, Caplan AB and Stiff JL: The visual analogue scale in the immediate postoperative period: intrasubject variability and correlation with a numeric scale. Anesth Analg 86: 102-106, 1998.

22. Ammar AS and Mahmoud KM: Effect of adding dexamethasone to bupivacaine on transversus abdominis plane block for abdominal hysterectomy: A prospective randomized controlled trial. Saudi J Anaesth 6: 229-233, 2012.

23. Bollag L, Richebe P, Siaulys M, Ortner CM, Gofeld M and Landau R: Effect of transversus abdominis plane block with and without clonidine on post-cesarean delivery wound hyperalgesia and pain. Reg Anesth Pain Med 37: 508-514, 2012.

24. Murphy DB, McCartney CJ and Chan VW: Novel analgesic adjuncts for brachial plexus block: A systematic review. Anesth Analg 90: 1122-1128, 2000.

25. Gupta A, Bodin L, Holmström B and Berggren L: A systematic review of the peripheral analgesic effects of intraarticular morphine. Anesth Analg 93: 761-770, 2001. 